\title{
Direct Comparison between the Angular Distributions of the Erythemal and Eye-Damaging UV Irradiances: A Pilot Study
}

\author{
P. Schouten ${ }^{\mathrm{a}, \mathrm{b}}$, A.V. Parisi ${ }^{\mathrm{b}}$.
}

${ }^{a}$ School of Engineering, Griffith University, Gold Coast, Australia 4222.

${ }^{\mathrm{b}}$ Centre for Rural and Remote Area Health, Faculty of Sciences, University of Southern Queensland, Toowoomba, Australia 4350.

Keywords: Solar UV, Ultraviolet, Angular, Erythemal, Spectroradiometer. 


\section{Abstract}

Several broadband ultraviolet (UV) radiation angular distribution investigations have been previously presented. As the biologically damaging effectiveness of $U V$ radiation is known to be wavelength dependent, it is necessary to expand this research into the distribution of the spectral $U V$. UV radiation is also susceptible to Rayleigh and Mie scattering processes, both of which are completely wavelength dependent. Additionally, the majority of previous measurements detailing the biologically damaging effect of spectral $U V$ radiation have been oriented with respect to the horizontal plane or in a plane directed towards the sun (sun-normal), with the irradiance weighted against action spectra formulated specifically for human skin and tissue. However, the human body consists of very few horizontal or sun-normal surfaces. Extending the previous research by measuring the distribution of the spectral irradiance across the sky for the complete terrestrial solar UV waveband and weighting it against erythemal, photoconjunctivital and photokeratital action spectra allowed for the analysis of the differences between the biologically effective irradiance $\left(U V_{B E}\right)$ values intercepted at different orientations and the effect of scattering processes upon the homogeneity of these $U V_{B E}$ distributions. It was established that under the local atmospheric environment, the distribution profile of the $U V_{B E}$ for each biological response was anisotropic, with the highest intensities generally intercepted at inclination angles situated between the horizontal and vertical planes along orientations closely coinciding with the sun-normal. A finding from this was that the angular distributions of the erythemal $U V_{B E}$ and the photoconjunctivital $U V_{B E}$ were different, due to the differential scattering between the shorter and longer UV wavelengths within the atmosphere. 


\section{Introduction}

Over the past thirty years, a number of investigations have been conducted focusing on the angular measurement and distribution of direct and diffuse solar UV radiation within varying atmospheric conditions. Employing a similar methodology as used in previous studies [1, 2, 3] Ireland and Sacher [4] conducted the most complete broadband solar UV angular distribution investigations made to date. The authors utilised a tripod-mounted narrow view radiometer to measure the angular distribution of the diffuse UV, visible and near infrared wavebands for different solar zenith angles (SZA) under near cloudless skies at a site in Lauder, New Zealand over a three-day period in early 1995. All radiometers used during the research had their input optic connected to a tube that limited their field of view to $5^{\circ}$ half-angle. During their analysis, Ireland and Sacher discovered that the angular distributions of the diffuse UV, visible and near infrared wavebands all exhibited anisotropic regimes, disagreeing with previous work $[5,6]$ that had postulated that isotropic distributions should be applied to certain solar wavebands.

The damaging effect of UV radiation on biological processes and tissue is related to photonic energy and is consequently dependent on wavelength. In order to accurately calculate the biologically damaging effect of UV radiation, the measured spectral UV irradiance must be weighted against a mathematical function known as an action spectrum [7]. An action spectrum quantifies the relative damaging effect or relative effectiveness of electromagnetic radiation to produce a particular biological response over a certain wavelength range [8]. In general, action spectra are determined by laboratory investigations performed on human, animal and plant test subjects. The biologically effective $\mathrm{UV}$ irradiance $\left(\mathrm{UV}_{\mathrm{BE}}\right)$ for a specific biological process with an 
action spectrum, $A(\lambda)$, is determined by applying this simple integral over the required UV wavelength range

$$
U V_{B E}=\int_{U V} S(\lambda) A(\lambda) d \lambda
$$

where $S(\lambda)$ is spectral irradiance in units of $\mathrm{W} \mathrm{m}^{-2} \mathrm{~nm}^{-1}$ [9]. In practice, the integral is substituted by a summation for spectral irradiances with a fixed wavelength range.

After collecting the results of almost sixty years of study by various researchers, the CIE [10] produced a definitive action spectrum (Figure 1) quantifying the erythemal (sunburning) effect of UV radiation on human skin. This spectrum shows that wavelengths below $298 \mathrm{~nm}$ are the most efficient at producing an erythemal response. From 298 to $328 \mathrm{~nm}$ the effectiveness decreases by close to three orders of magnitude. Wavelengths increasing from $328 \mathrm{~nm}$ display a steady reduction in erythemal effectiveness by approximately four orders of magnitude compared to the effectiveness at $298 \mathrm{~nm}$.

Excessive exposure of the eye to UV radiation causes the onset of photoconjunctivitis, an inflammation of the conjunctiva, the soft tissue covering the eyelid and some of the eyeball. The CIE [11] developed a model quantifying the spectral responsivity of photoconjunctivitis (Figure 1) using previous work compiled over a forty-year period. From $220 \mathrm{~nm}$ the photoconjunctivital effectiveness has a gradual reduction before sharply increasing at $235 \mathrm{~nm}$ until reaching the maximal response occurring at 260 nm. After the maxima, the photoconjunctivital response rapidly decreases to zero response at $310 \mathrm{~nm}$, and hence no response is seen in the UVA waveband. 
Photokeratitis is a swelling of the cornea that occurs after excessive exposure to UV radiation generally from artificial sources such as welding arcs, mercury lamps, laser beams and reflected UV from sand and snow. After analysing the data assimilated by numerous research groups on various live test subjects including rabbits, primates and humans, the CIE [12] delivered a recommended action spectrum for photokeratitis (Figure 1). The maximum response for photokeratitis is located at $288 \mathrm{~nm}$, with no response in the UVA. In general, the responsivity decreases more rapidly away from the maxima at longer wavelengths in comparison to the shorter wavelengths.

Following on from the broadband analysis performed by McKenzie, Paulin and Kotkamp [13] limited spectral work was undertaken by Parisi and Kimlin [9] who measured the horizontal and sun-normal biologically damaging spectral UV irradiances at a Southern Hemisphere site. All measurements were made using a mobile spectroradiometer over two clear-sky days in late winter and early spring. The results showed that a surface oriented in a sun-normal direction could receive up to $27 \%$ higher erythemal UV exposure in comparison to a horizontal plane. Also, for the sun-normal orientation a reduction in diffuse UV irradiance was observed when compared to the horizontal orientation. This equated to a decrease in the damaging UV exposure produced by the diffuse component in the sun-normal orientation.

Parisi, Kimlin and Turnbull [14] extended this research by investigating the effect of tree shade on the solar UV irradiance incident on horizontal and sun-normal surfaces. A spectroradiometer was employed to measure irradiances for horizontal and sunnormal orientations under trees with medium and thin canopies with measurements taken at the centre, edge and trunk of each tree. Further studies of how diffuse 
radiation can potentially influence human eye damage in both full sun and tree shade conditions [15] and a year long analysis of the angular distribution of biologically damaging solar UV radiation under public shade structures at sports fields and public venues [16] have also been detailed. Continuing on from these studies, the most recent examination into the angular distribution of solar UV radiation was performed by Koepke and Mech [17] who analysed the influence of SZA, albedo and cloud coverage upon erythemal irradiance incident on tilted surfaces. From this work it was found that the largest clear sky erythemal irradiance values on an inclined surface are most likely to be measured during high SZA conditions and are enhanced by surrounding high ground albedo.

The vast majority of measurements detailing biologically damaging UV radiation are oriented with respect to some horizontal surface. The spectral irradiance is weighted against action spectra formulated specifically for human skin and tissue, but the surface of the human physique consists of very few horizontal surfaces. So the angular distribution of UV radiation must be accurately quantified in order for future research to create a truly practical model detailing the effect of UV on the entire surface of the human body.

The only known study into the angular distribution of the solar spectral UV and erythemal UV across a large part of the terrestrial UV waveband has been performed in Japan by Kawanishi [18] who developed a rudimentary distribution of the spectral ultraviolet radiation for sections of the sky. In this study, the distribution was weighted against only the erythemal action spectrum and no other action spectra were considered. 
To the authors' knowledge, apart from the preliminary work detailed in [18], only broadband angular UV radiation distribution investigations have been made to this point in time. Consequently, spectral angular distributions are required, as the biologically damaging effectiveness of UV is completely wavelength dependent. Additionally, UV radiation is also susceptible to Rayleigh and Mie scattering processes, both of which are wavelength dependent. Some spectral analysis has been compiled for specific directions and inclinations, but it has yet to encompass the entire hemispherical domain, which is necessary, as the diffuse UV irradiance is incident from all angles and directions. Consequently, this paper addresses this definite need for a study that accurately quantifies the angular distribution of the spectral solar UV spectrum at different SZA by reporting on a direct comparison between the angular distribution of the erythemal and eye-damaging UV irradiances.

\section{Materials and Methods}

\section{Equipment Setup and Usage Summary}

The angular distribution of the solar UV spectrum was investigated for multiple combinations of seven inclinations and eight directions using a wide view sampling approach to map the distribution of the solar UV spectrum around a hemispherical domain encompassing the sky. Every field-based spectral UV measurement trial was undertaken at the University of Southern Queensland's Toowoomba campus ( $27^{\circ} 36^{\prime}$ $\mathrm{S}, 151^{\circ} 55^{\prime}$ E) utilising transportable spectroradiometric instrumentation fitted with a specialised angular sweep apparatus. A picture of the spectroradiometer along with the angular sweep apparatus is depicted in Figure 2. The spectral UV measurements were taken under both clear sky and partially cloudy conditions in an open outdoor environment free from any natural or artificial obstructions. For the remainder of this 
manuscript the trial carried out under partially cloudy sky conditions will be referred to as trial 1 and the trial performed under clear sky conditions will be referred to as trial 2. Biologically effective angular irradiance distributions were also determined from the spectral UV data.

The transportable scanning spectroradiometer employed a diffuser input optic (type D6, Bentham Instruments, Reading, UK) capable of collecting spectral data ranging from 280 to $400 \mathrm{~nm}$ with wavelength dispersal provided by a double holographic grating monochromator (model DH10, Jobin Yvon, France) with 1200 lines/mm gratings. Detection of the irradiances was performed by a photomultiplier tube (model R212, Hamamatsu Co., Japan) maintained at a temperature of $15.0 \pm 0.5^{\circ} \mathrm{C}$ by a Peltier cell controller. A custom built angular sweep apparatus was attached to the diffuser, which allowed for measurements to be made along any axis and at any angle from horizontal to vertical. The cosine error of the diffuser has been measured to be no greater than $1 \%$ as specified by the manufacturer [19].

Before every scanning session, the spectroradiometer was wavelength calibrated against mercury UV spectral lines and absolute irradiance calibrated against a $250 \mathrm{~W}$ secondary standard quartz tungsten halogen lamp (SSL) operating at a current of $9.500 \pm 0.005$ A d.c. with calibration traceable to a primary standard located at the CSIRO National Standards Laboratory, Lindfield. The SSL current was via a regulated power supply (Model PD36 20AD, Kenwood) that was monitored by a calibrated multimeter (MX 56 Metrix). According to the CSIRO [20] this primary standard has an estimated uncertainty of. $\pm 3.0 \%$ at $240 \mathrm{~nm}$ that decreases linearly with increasing wavelength to $\pm 2.0 \%$ at $350 \mathrm{~nm}$ and decreases further with increasing 
wavelength to $\pm 1.1 \%$ at $550 \mathrm{~nm}$. In addition to this, it is known that an error of approximately $\pm 3.0 \%$ is inherited in the calibration transfer between the primary to secondary standard. Also, the variations in the spectral irradiance measured by the spectroradiometer are known to be in the order of $\pm 5 \%$ [21].

For the absolute irradiance calibration sessions with the spectroradiometer, the SSL was mounted in a custom-built housing above the diffuser. This housing was specifically designed so that the interference of stray light was kept to an absolute minimum and also to keep the SSL at an adequate temperature via an external cooling device. The responsivity of the spectroradiometer was determined by scanning the standard lamp at the required lamp current and voltage settings and lamp to aperture distance to sustain utmost repeatability. Following the irradiance calibration, a dark count (DK) scan was performed where the DK is defined as the measurement of randomised electronic noise within the spectroradiometer. The DK scan was made by performing a 'clean' scan by ensuring that absolutely no radiation was incident on the diffuser. Three DK scan cycles were taken for each measurement campaign.

\section{Measurement Campaign Specification}

The angular distribution of the solar UV spectrum over various inclination and direction combinations was recorded using the mobile scanning spectroradiometer for both near clear sky (trial 1) and partially cloudy (trial 2) conditions. The measurements were taken out in an open outdoors environment (a football field) free from any type of natural or artificial obstruction. The surface of this field was predominantly grass with an estimated albedo of $1.9 \% \pm 0.15 \%$. This albedo measurement was made using an IL1400 broadband meter ('A' Series, International 
Light, Newburyport, MA) calibrated directly to the spectroradiometer used in the measurement trials.

For each of the two cloud coverage conditions, measurements were taken on separate days across an approximate one hour time period as quickly as possible generally commencing in the mid morning ending in the early afternoon during the winter months of 2005. Over this time the SZA ranged over $60^{\circ}$ to $48^{\circ}$ on the days of the measurements. The determination of the SZA was made using data available from the US Naval Observatory Sun Azimuth and Altitude Table Internet site located at http://aa.usno.navy.mil/data/docs/AltAz.html.

Clear sky measurements were taken on occasions when no visible cloud was present in the surrounding skies. Distant low level cloud seen low on the outer horizon during some measurements was disregarded. The percentage of cloud coverage present during the measurement trials reached a maximum of $34 \%$ and a minimum of $1 \%$. These cloud coverage amounts were determined from processed sky data accumulated by a total sky imager system (TSI-440, Yankee Environmental Systems, MA, USA) currently maintained at the University of Southern Queensland's Toowoomba campus approximately 200 metres away from the measurement site. The TSI-440 has been found to have an uncertainty of approximately $\pm 10 \%$ at least $95 \%$ of the time during its operation [22].

As the measurements were carried out in 2005 , over the entirety of the solar measurement phase local ozone levels and aerosol index data over Toowoomba was collected from the Goddard Space Flight Centre Total Ozone Spectrophotometer 
(GSFC-TOMS)

information

page

found

at

http://toms.gsfc.nasa.gov/eptoms/ep_ovplist_a.html. These readings were taken from a daily pass by the TOMS satellite at 11:15 am local time at a surveillance resolution of $50 \mathrm{~km} \times 50 \mathrm{~km}$ at nadir and $100 \mathrm{~km}$ x $100 \mathrm{~km}$ average [23]. Specific values for trial 1 and trial 2 can be seen in Table 1. At this time the TOMS instrumentation had a latitude dependent calibration error for the ozone values of between -2 to $4 \%$. The aerosol index (AI) values as provided in Table 1 are calculated from the ratio between measured backscattered UV radiation from the Earth's atmosphere at $360 \mathrm{~nm}$ by the TOMS satellite and the modelled backscattered UV radiation predicted for a pure Rayleigh atmosphere at $360 \mathrm{~nm}$ [24]. The AI is negative for aerosols with poor absorption and is positive for aerosols with good absorption.

Before each scan, the spectroradiometer was levelled to ensure that the diffuser was aligned on a horizontal plane. Spectral data was scanned in $1 \mathrm{~nm}$ increments over a wavelength range extending from 280 to $400 \mathrm{~nm}$, although for most scans a useable irradiance signal above the noise was not obtained until at least $295 \mathrm{~nm}$. Each scan took approximately $45 \mathrm{~s}$ to complete. For both trial 1 and trial 2 scans were halted when rapid changes in cloud coverage occurred and were resumed once all cloud had moved over and away from the solar disk.

The solar UV spectrum was measured for seven different inclination angles $(\delta)$ in steps of $15^{\circ}$ over a range spanning from $0^{\circ}$ through to $90^{\circ}$ with respect to the horizontal plane for eight different direction angles $(\omega)$ working in $45^{\circ}$ increments from geographical north, namely north, north-east, east, south-east, south, south-west, west and north-west. This collective of measurements generated a hemispherical 
measurement domain, which is depicted in Figure 3. The $90^{\circ}$ scans were made in conjunction with each azimuth and inclination combination series in order to compare the angular measurements against a closely timed measurement aligned in parallel to the Earth's surface plane. A compass was used to align the angular sweep apparatus to the particular direction under analysis. Due to parallax error occurring during the use of the compass, it is estimated that an inaccuracy of $\pm 2^{\circ}$ became inherent with each directional orientation. Less than $50 \mathrm{~s}$ was required for each change in direction and less then $15 \mathrm{~s}$ was needed for each change in inclination angle as the angular sweep apparatus had the required angles marked on its axial pivot. After the solar measurement phase was completed, Microsoft Excel (versions 97 and 2002) was used to produce the graphical representations of the collected data.

\section{Results}

Table 1 details the various environmental factors associated with the two trials reported in this manuscript. Figure 4 displays the spectral UV irradiance angular distribution for trial 1 in the upper graph and trial 2 in the lower graph. The spectral irradiance data displayed in Figure 4 is not given relative to a single measurement and has not been normalised or averaged. Each UV spectrum is a singular unweighted irradiance scan measured by the spectroradiometer at a particular instant for an exact point within the hemispherical domain measured at a given time throughout the approximate 1 hour measurement campaign time span. In each graph the $\mathrm{x}$-axis represents the wavelength in nanometres and the z-axis gives the spectral irradiance in units of Watts per square metre per nanometre. The y-axis illustrates the seven different inclination angles in steps of $15^{\circ}$ over a $90{ }^{\circ}$ range with respect to the horizontal plane for the eight direction angles moving in $45^{\circ}$ increments from 
geographical south to geographical south-east. As an example, between the interval 0 $\mathrm{N}$ and $0 \mathrm{NE}$, the spectral data is presented for the inclination and direction combinations of $0{ }^{\circ} \mathrm{N}, 15^{\circ} \mathrm{N}, 30^{\circ} \mathrm{N}, 45^{\circ} \mathrm{N}, 60^{\circ} \mathrm{N}, 75^{\circ} \mathrm{N}$ and $90^{\circ} \mathrm{N}$. This format remains similar for all the other azimuths. For all of the inclination and direction angle combinations the spectral irradiance increased with increasing wavelength from the cut-off wavelength of $295 \mathrm{~nm}$. The troughs seen at the same wavelengths throughout the spectral irradiances are caused by the absorption of UV radiation by various molecular constituents within the Sun's atmosphere and the Earth's atmosphere.

The spectral $U_{\mathrm{BE}}$ distributions corresponding to trial 1 and trial 2 for the erythemal, photoconjunctivital and photokeratital responses are illustrated in the form of a threedimensional contour map in Figure 5. In these contour maps the three axes represent the same quantities as detailed before for Figure 4. These contour maps show that in general under both clear and cloudy conditions for SZAs between $48^{\circ}$ and $60^{\circ}$, the highest $\mathrm{UV}_{\mathrm{BE}}$ levels were received at inclination angles between $15^{\circ}$ and $75^{\circ}$ either near or along the north or north-west directions. In addition, the contour maps display the disparity of $\mathrm{UV}_{\mathrm{BE}}$ levels across the different inclination angle and direction combinations that reveal the anisotropic distribution of the $\mathrm{UV}_{\mathrm{BE}}$ for each of the various biological responses.

For trial 1 and trial 2, Figure 6 and Figure 7 respectively compare the distribution of the erythemal $\mathrm{UV}_{\mathrm{BE}}$ to the distributions of the $\mathrm{UV}_{\mathrm{BE}}$ for photoconjunctivitis and photokeratitis across the hemispherical domain. These $\mathrm{UV}_{\mathrm{BE}}$ measurements were selected due to the differences in the responsive wavelengths of the respective action 
spectra. In each graph, the $\mathrm{x}$-axis displays the particular inclination and direction combinations sampled across the sky. The dimensionless y-axis illustrates the ratio between each of the $\mathrm{UV}_{\mathrm{BE}}$ values intercepted over the sky for a specific response to the highest $\mathrm{UV}_{\mathrm{BE}}$ value measured for that particular response. This normalisation of the $U V_{B E}$ was performed in order to magnify certain small changes in the $U V_{B E}$ between the erythemal and eye-damaging responses that could not be clearly seen by analysing the original $\mathrm{UV}_{\mathrm{BE}}$ irradiances. The error bars are $\pm 10 \%$ and are calculated as the accumulation of the $\pm 5 \%$ estimated error in each scan of the spectroradiometer. The error associated with the calibration lamp is not included here as this error remains the same for each scan and the aim of this analysis is to find a relative difference between scans.

\section{Discussion}

Across all sections of the sky, the distribution of the solar spectral irradiance was found to be approximately homogeneous within the UVB until reaching the UVA where the distribution progressively degenerated into anisotropy. The main cause of this was considered to be due to the dissimilar levels of Rayleigh scattering between the shorter UVB wavelengths and the longer UVA wavelengths, which leads to a far more uniform distribution of the UVB across the sky in comparison to the UVA. In addition, generally there is a much higher level of UVA present in the atmosphere in comparison to the UVB at high SZA, particularly during the hours of the afternoon when SZA begins in increase over time and as a result more UVB is absorbed by ozone. This large ratio between UVA and UVB wavelengths would have been another influencing factor affecting the anisotropic distribution of the spectral UVA as seen in 
both trial 1 and trial 2 which both extended from late morning to the early afternoon spanning across an SZA range of approximately $10^{\circ}$.

The anisotropic distribution of the spectral irradiance seen in these trials can not only be associated to Rayleigh scattering. Scattering caused by natural and industrial aerosols and cloud cover also has an influence upon incoming solar UVB and UVA photons. Additionally, the position of the sun within the east-west plane of the sky contributes in part to the anisotropic profile of the spectral solar UV. Each trial was undertaken during either the mid to late morning or the early afternoon. During these times of the day, at the measurement location, the sun transverses across the north to the north-west as it makes its way out towards the west in the late afternoon, so the measurements made in these directions were intercepting the solar UV irradiance at either a near or exact sun-normal orientation, which accounts for the significantly higher spectral irradiances generally seen across the directions moving from the west to the north in comparison to the directions spanning around from the north-east to the south-west. These directions running from the north-east to the south-west had no clear direct view of the sun and consequently were receiving only the localised diffuse UV irradiance.

Trial 1 taken during slightly cloudy conditions exhibited moderately higher spectral irradiances in comparison to those intercepted during trial 2 taken under completely cloud free conditions during the same month for a similar SZA range. As some high cirrus cloud was seen moving close to the solar disk during trial 1 it could be a possibility that some solar UV enhancement came into effect during the measurements. For example, in trial 1, the spectral intensity seen at the $45^{\circ} \mathrm{NW}$ 
inclination angle and direction reached a maxima of approximately $1.4 \mathrm{~W} \mathrm{~m}^{-2} \mathrm{~nm}^{-1}$ at $400 \mathrm{~nm}$, while for trial 2 the spectral intensity of the corresponding inclination angle and direction reached a maxima of approximately $1.7 \mathrm{~W} \mathrm{~m}^{-2} \mathrm{~nm}^{-1}$ at $400 \mathrm{~nm}$, an increase of roughly $21 \%$, which is within the limits of $25 \pm 23 \%$ for UVA enhancement as defined by [25] and above the total uncertainty inherent within the spectroradiometer. Additionally, a lower level of ozone was also present during the trial 1 measurements (239 DU) in comparison to the trial 2 measurements (286 DU) which could have had a measureable influence on the higher irradiance intensities detected in trial 1 .

For trial 1 and trial 2, as seen in Figure 6, only very slight differences were seen between the erythemal and photokeratital $\mathrm{UV}_{\mathrm{BE}}$ distributions across the hemispherical domain. It is unclear whether these differences are genuine or are the result of the innate irradiance variation within the spectroradiometer as they all fall within the $10 \%$ level of uncertainty. However, large variations extending across the majority of the inclination and direction combinations often exceeding the $10 \%$ uncertainty threshold were seen to exist between the erythemal and photoconjunctivital $\mathrm{UV}_{\mathrm{BE}}$ distributions. It should be noted that the maximum irradiance did occur at the $90^{\circ} \mathrm{SE}$ position for trial 1 and the corresponding trial 2 measurement in this position was also relatively high. As detailed in the Materials and Methods section, the $90^{\circ}$ angle as defined within the hemispherical domain is effectively a measurement parallel to the Earth's surface plane and is not effectively pointing in the SE direction. So at the time that this measurement was made in both trial 1 and trial 2 the Sun would have been either very close to or at its highest point in the sky in order for the highest irradiance value to be measured. 
As previously mentioned, longer wavelengths, such as those near or within the UVA, are less sensitive to Rayleigh scattering than the shorter UVB wavelengths, resulting in the scattering of the longer wavelengths being less uniform across the sky, while the scattering of the shorter wavelengths remaining approximately isotropic in comparison. This explains the differences between the erythemal and photoconjunctivital $\mathrm{UV}_{\mathrm{BE}}$ distributions and the similarities between the erythemal and photokeratital $\mathrm{UV}_{\mathrm{BE}}$ distributions. The photoconjunctivital action spectrum is only effective within the UVB until a cut-off at $310 \mathrm{~nm}$. However, the photokeratital action spectrum extends out closer to the UVA until a cut-off at $316 \mathrm{~nm}$, while the erythemal action spectrum remains effective through the UVB and across the entirety of the UVA. So the distribution of $\mathrm{UV}_{\mathrm{BE}}$ for the erythemal and photokeratital responses is influenced to a lesser degree by scattering in comparison to the $U V_{\mathrm{BE}}$ for the photoconjunctivial response.

Under trees, purpose-built shade structures and hats, the diffuse solar UV component constitutes the largest proportion of total human UV exposure and hence provides the greatest biological threat in these environments. The results taken from this research will provide an improved characterisation of the spectral angular distribution of the diffuse solar UV component, which should assist in the better design of future UV minimisation strategies. Further research will be required to quantify the effect of cloud cover, aerosols, ozone and SZA less than $48^{\circ}$ upon the distribution of the spectral UV. This data could be used to synthesise a model that would determine the variations throughout the year of $\mathrm{UV}_{\mathrm{BE}}$ exposure under particular shade settings. 
Acknowledgments:

The authors acknowledge the technical staff of the USQ physics discipline and the Sciences workshop for their assistance in this project. 


\section{References}

[1] J.E. Burt, F.M. Luther. Effect of receiver orientation on erythema dose. Photochem. Photobiol. 29 (1979) 85-91.

[2] A. Dahlback, J. Moan. Annual exposures to carcinogenic radiation from the sun at different latitudes and amplification factors related to ozone depletion. The use of different geometrical representations of the skin surface receiving the ultraviolet radiation. Photochem. Photobiol. 52 (1990) 1025-1028.

[3] A.V. Parisi, C.F. Wong. A dosimetric technique for the measurement of ultraviolet radiation exposure to plants, Photochem. Photobiol. 60 (1994) 471-474.

[4] W. Ireland, R. Sacher. The angular distribution of solar ultraviolet, visible and near-infrared radiation from cloudless skies. Photochem. Photobiol. 63 (1996) 483486.

[5] J.E. Frederick. Yearly review. Ultraviolet sunlight reaching the Earth's surface: A review of recent research. Photochem. Photobiol. 57 (1993) 175-178.

[6] R.G. Grainger, R.E. Basher, R.L. McKenzie. UV-B Robertson-Berger meter characterisation and field calibration. Applied Optics, 32 (1993) 343-349.

[7] M.M. Caldwell, L.B. Camp, C.W. Warner, S.D. Flint. Action spectra and their key role in assessing biological consequences of solar UV radiation, Stratospheric Ozone Reduction, Solar Ultraviolet Radiation and Plant Life, R.C. Worrest, M.M. Caldwell (eds.) (1986) Springer, Heidelberg.

[8] J.C.F. Wong, A.V. Parisi. 1996, Measurement of UVA exposure to solar radiation. Photochem. Photobiol. 63(6) (1996) 807-810.

[9] A.V. Parisi, M.G. Kimlin. Horizontal and sun-normal biologically effective ultraviolet irradiances. Photochem. Photobiol. 53(1-3) (1999) 70-74. 
[10] CIE (International Commission on Illumination). A reference action spectrum for ultraviolet induced erythema in human skin. CIE-Journal. 5(1) (1987) 17-22.

[11] CIE (International Commission on Illumination). Photoconjunctivitis. CIEJournal. 5(1) (1986) 24-28.

[12] CIE (International Commission on Illumination). Photokeratitis. CIE- Journal. 5(1) (1986) 19-23.

[13] R.L. McKenzie, K.J. Paulin, M. Kotkamp. Erythemal UV irradiances at Lauder, New Zealand: Relationship between horizontal and normal incidence. Photochem. Photobiol. 66 (1997) 683-689.

[14] A.V. Parisi, M.G. Kimlin, D. Turnbull. Spectral shade ratios on horizontal and sun normal surfaces for single trees and relatively cloud free sky. J. Photochem. Photobiol. B:Biol. 65 (2001) 151-156.

[15] A.V. Parisi, A. Green, M.G. Kimlin. Diffuse Solar UV Radiation and Implications for preventing Human Eye Damage. Photochem. Photobiol. 73(2) (2001) 135-139.

[16] D.J. Turnbull, A.V. Parisi. Annual variation of the angular distribution of UV beneath public shade structures. J. Photochem. Photobiol. B:Biol, 76(1-3) (2004) 4147.

[17] P. Koepke, M. Mech. UV irradiance on arbitrarily oriented surfaces: variation with atmospheric and ground properties. Theor. Appl. Climatol. 81 (2005) 25-32.

[18] Kawanishi, T. in Proceedings of SPIE Vol. 5886 Ultraviolet Ground- and Spacebased Measurements, Models and Effects V, eds. G. Bernhard, J.R. Slusser, J.R. Herman, W. Gao (SPIE, Belligham, WA, 2005).

[19] Bentham Instruments n.d., Cosine Response Diffusers, United Kingdom, viewed 8 October 2010, < http://www.bentham.co.uk/pdf/Cosine\%20Diffusers.pdf> 
[20] CSIRO (Commonwealth Scientific and Industrial Research Organisation) 1997, National Measurement Laboratory Measurement Report On One Tungsten Halogen Lamp No. M28623, CSIRO, Lindfield, Australia.

[21] C.F. Wong, S. Toomey, R.A. Fleming, B.W. Thomas. UV-B radiometry and dosimetry for solar measurements. Health Phys. 68 (1995) 175-184.

[22] J.M. Sabburg, C.N. Long. Improved sky imaging for studies of enhanced UV irradiance. Atmos. Chem. Phys. 4 (2004) 2543-2552.

[23] A.V. Parisi, J. Sabburg, M.G. Kimlin, Scattered and Filtered Solar UV Measurements, Kluwer Academic Publishers, 2004.

[24] NASA 2006, Aerosol Data, United States, viewed 8 October 2010, < http://jwocky.gsfc.nasa.gov/aerosols/AI_definition/ai_ep_definition.pdf> [25] J.M. Sabburg, A.V. Parisi, M.G. Kimlin. Enhanced spectral UV irradiance: A 1 year preliminary study. Atmos. Res. 66 (2003) 261-272. 
Table 1: Specific measurement trial details.

\begin{tabular}{|l|l|l|l|l|l|l|l|l|}
\hline Trial Name & \multicolumn{1}{|c|}{ Date } & $\begin{array}{c}\text { SZA Range } \\
\left.\text { Over Trial ( }{ }^{\circ}\right)\end{array}$ & $\begin{array}{c}\text { Solar Azimuth } \\
\left.\text { Range } \mathbf{(}^{(}\right)\end{array}$ & $\begin{array}{c}\text { Percent Cloud } \\
\text { Coverage } \\
\text { (Minimum to } \\
\text { Maximum) }\end{array}$ & $\begin{array}{c}\text { Local Aerosol } \\
\text { Index }\end{array}$ & $\begin{array}{c}\text { UV Index } \\
\text { Local Ozone } \\
\text { Level (Dobson } \\
\text { Units) }\end{array}$ & $\begin{array}{c}\text { Cloud } \\
\text { Coverage } \\
\text { Description }\end{array}$ \\
\hline Trial 1 & 8 July 2005 & 60 to 51 & 41 to 10 & 1 to 34 & -0.5 & 12 & $\begin{array}{l}239 \\
\text { Partially cloudy some cloud } \\
\text { visible near the } \\
\text { solar disk }\end{array}$ \\
\hline Trial 2 & 26 July 2005 & 58 to 48 & 39 to 14 & 1 to 2 & -2.8 & 12 & $\begin{array}{l}\text { Clear sky. No } \\
\text { cloud near the } \\
\text { solar disk }\end{array}$ \\
\hline
\end{tabular}




\section{FIGURE CAPTIONS}

Figure 1: The erythemal action spectrum (CIE [10]), the photoconjunctivital action spectrum (CIE [11]) and the photokeratital action spectrum (CIE [12]).

Figure 2: The configuration of the mobile scanning spectroradiometer in operation in the field.

Figure 3: The hemispherical measurement environment where $\delta$ denotes the inclination angle, $\omega$ defines the direction/azimuth angle and $\psi$ gives the SZA. The detector is located at the origin $\mathrm{O}$.

Figure 4: The distribution of spectral irradiance spanning over the hemispherical domain for A) trial 1 and B) trial 2. The inclination angle is measured in degrees.

Figure 5: Spectral distributions of the erythemal, photoconjunctivital and photokeratital $\mathrm{UV}_{\mathrm{BE}}$ across the hemispherical domain shown for trial 1 in charts $\mathrm{Ai}$ ), Bi) and $\mathrm{Ci}$ ) respectively and for trial 2 in charts Aii), Bii) and Cii).

Figure 6: Comparison of the normalised values corresponding to trial $1 \mathrm{for} \mathrm{Ai}$ ) the erythemal and photoconjunctivital response and $\mathrm{Bi}$ ) the erythemal and photokeratital response. The inclination angle is measured in degrees.

Figure 7: Comparison of the normalised values corresponding to trial 2 for Aii) the erythemal and photoconjunctivital response and Bii) the erythemal and photokeratital response. The inclination angle is measured in degrees. 


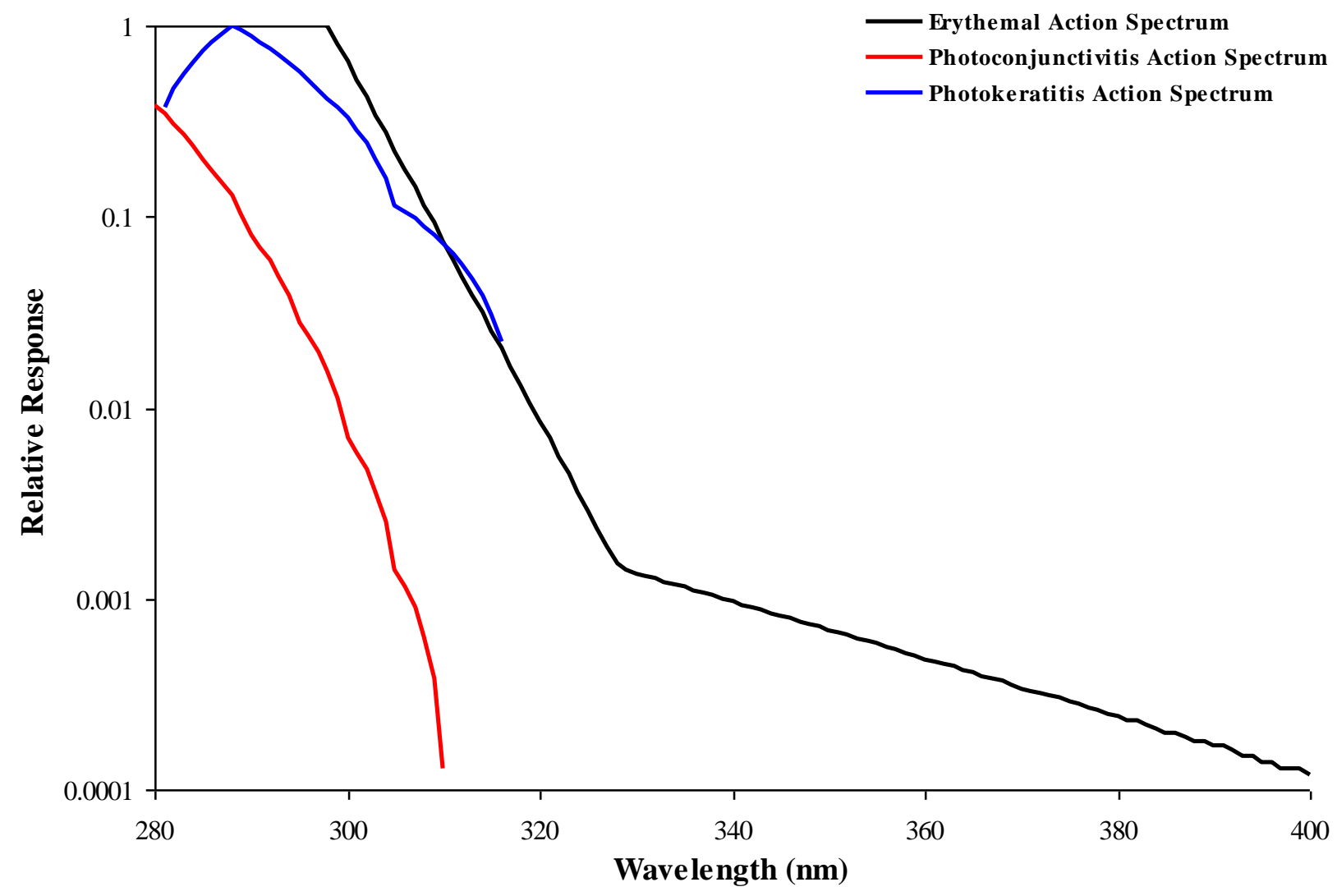

Figure 1: The erythemal action spectrum (CIE [10]), the photoconjunctivital action spectrum (CIE [11]) and the photokeratital action spectrum (CIE [12]). 


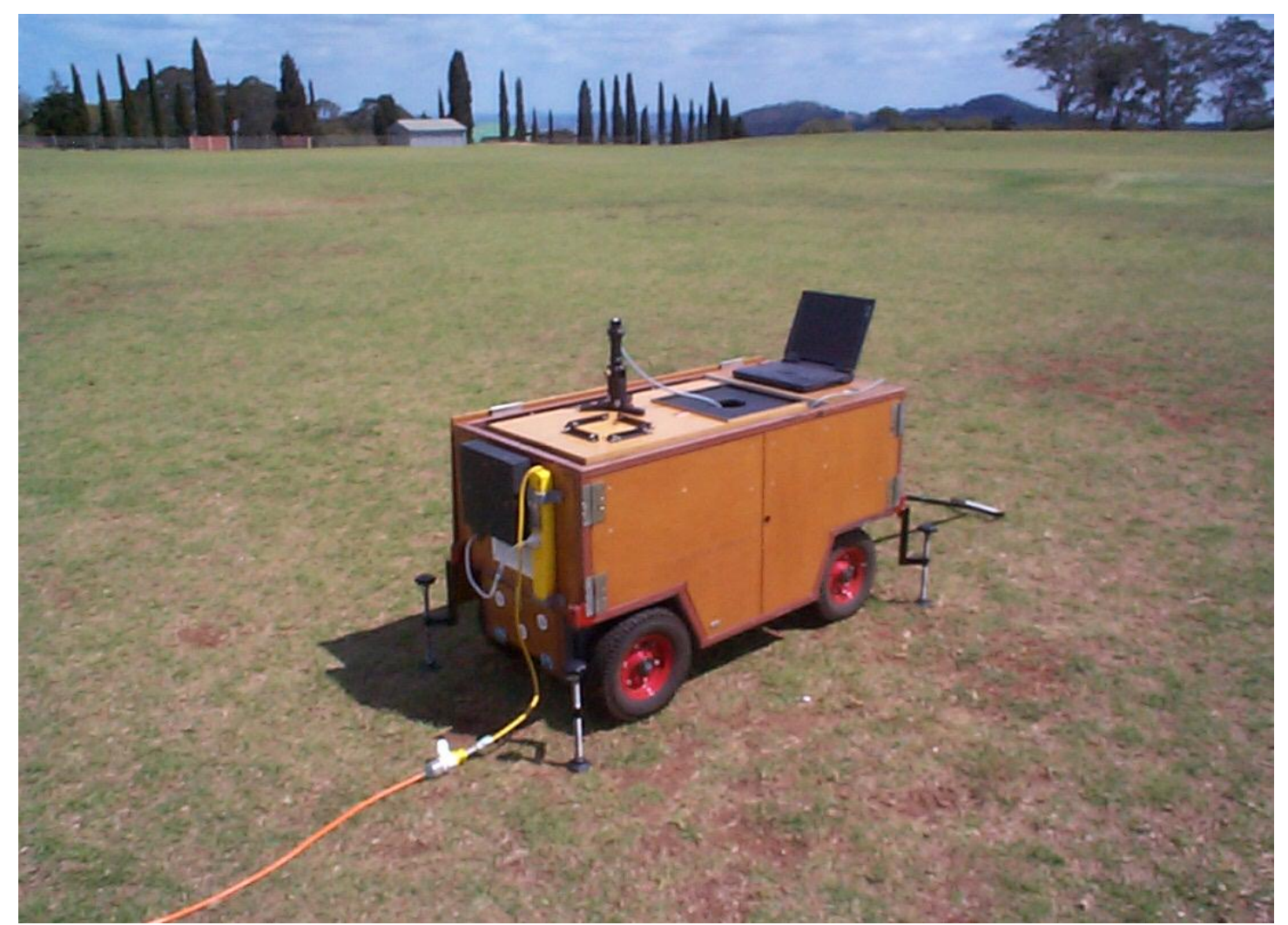

Figure 2: The configuration of the mobile scanning spectroradiometer in operation in the field. 


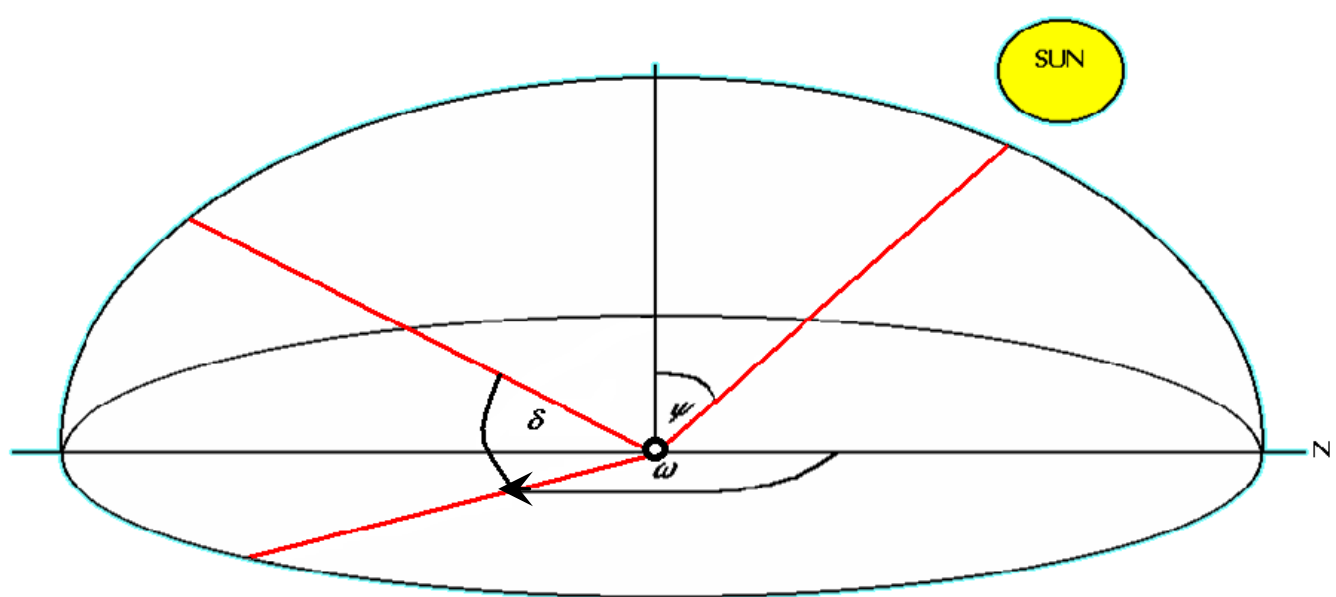

Figure 3: The hemispherical measurement environment where $\delta$ denotes the inclination angle, $\omega$ defines the direction/azimuth angle and $\psi$ gives the SZA. The detector is located at the origin $\mathrm{O}$. 


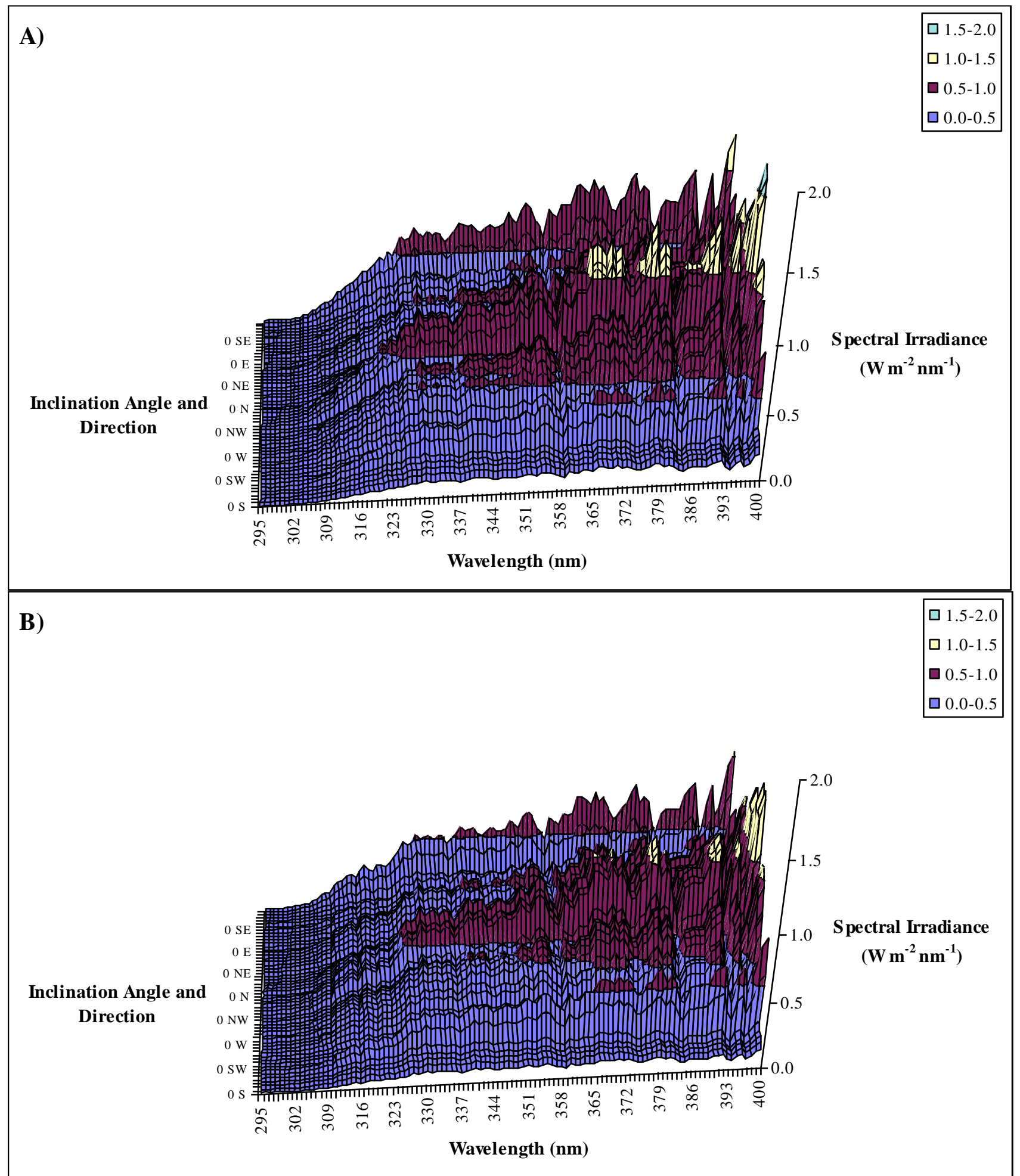

Figure 4: The distribution of spectral irradiance spanning over the hemispherical domain for A) trial 1 and B) trial 2. The inclination angle is measured in degrees. 


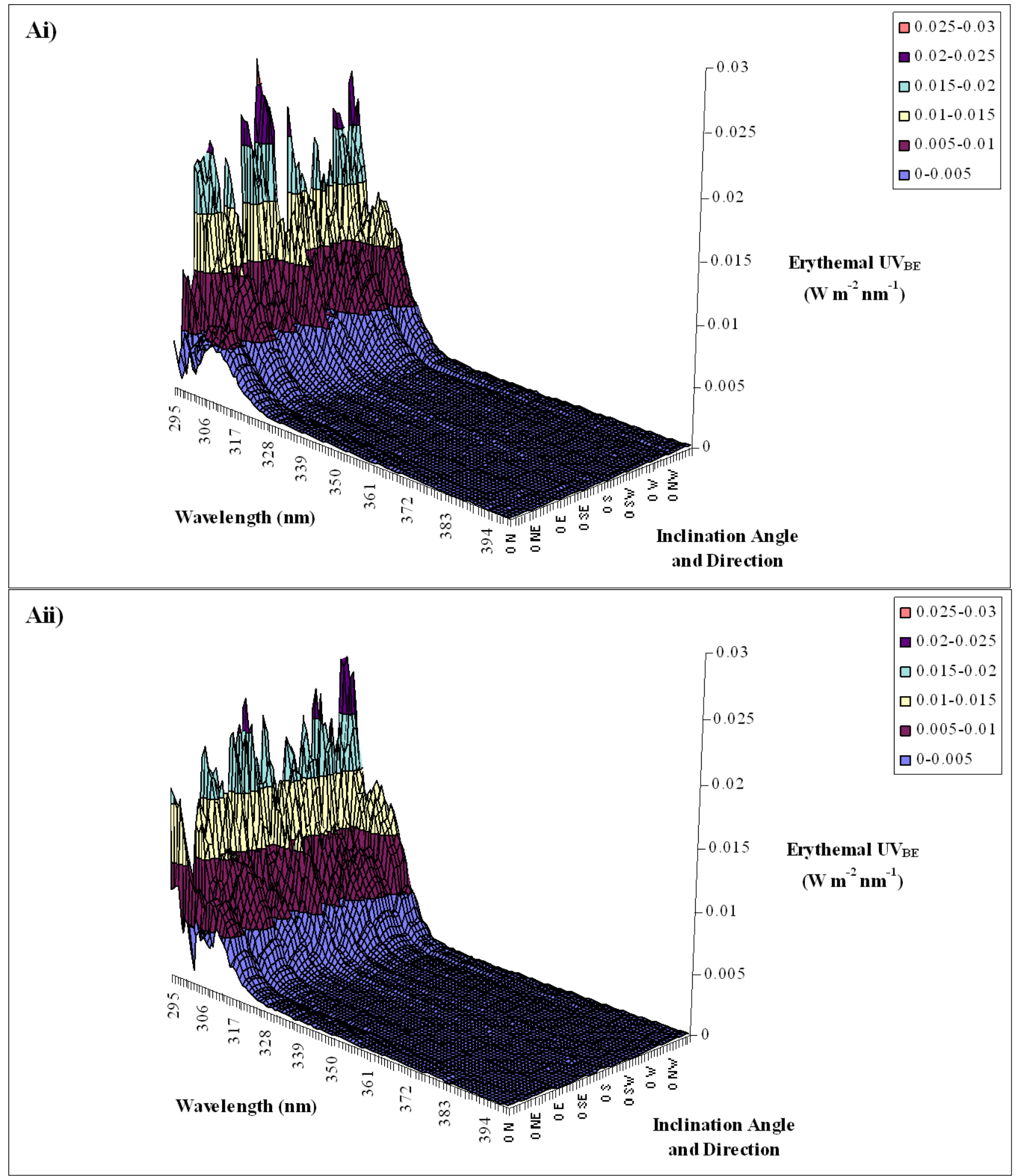




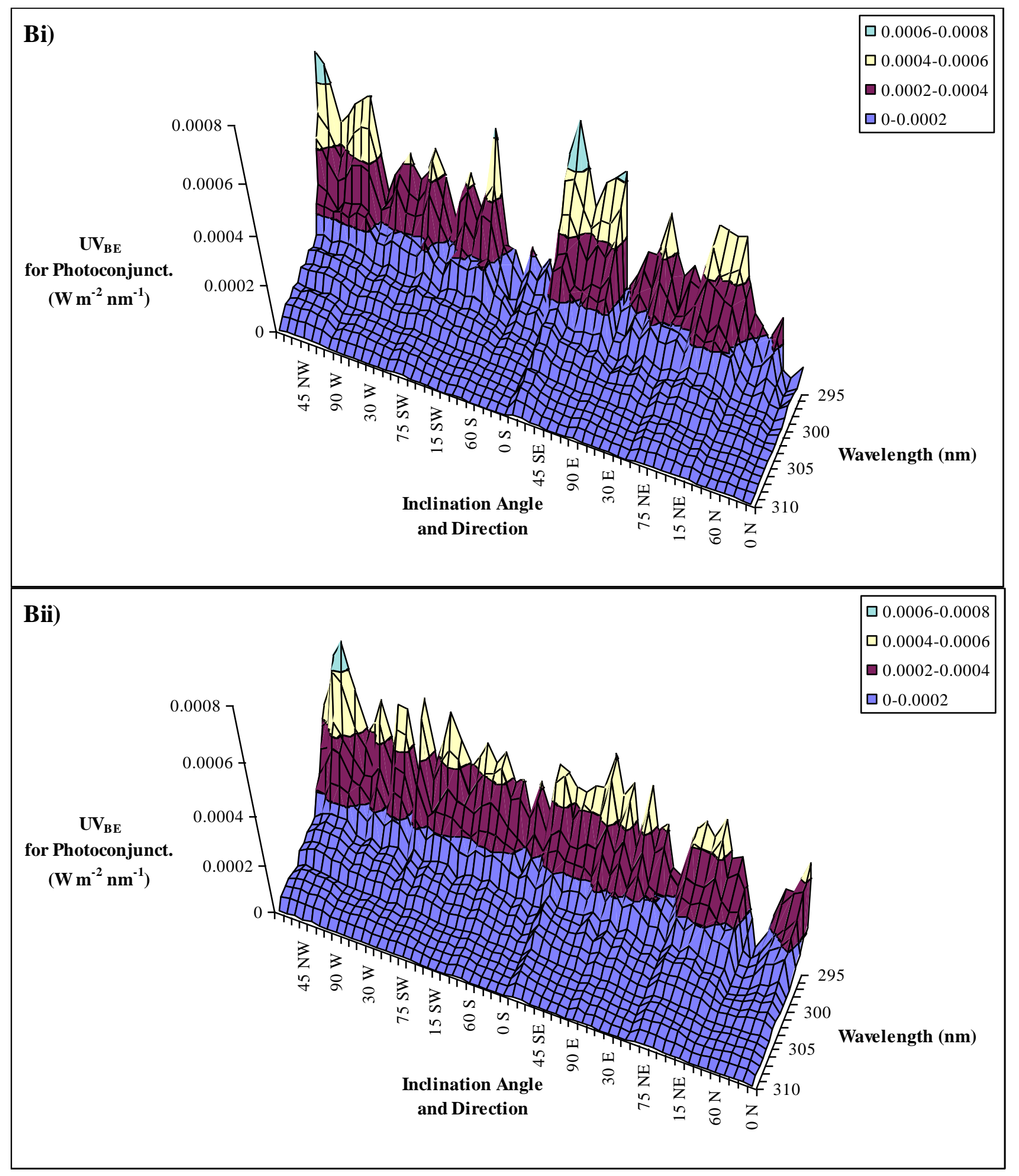




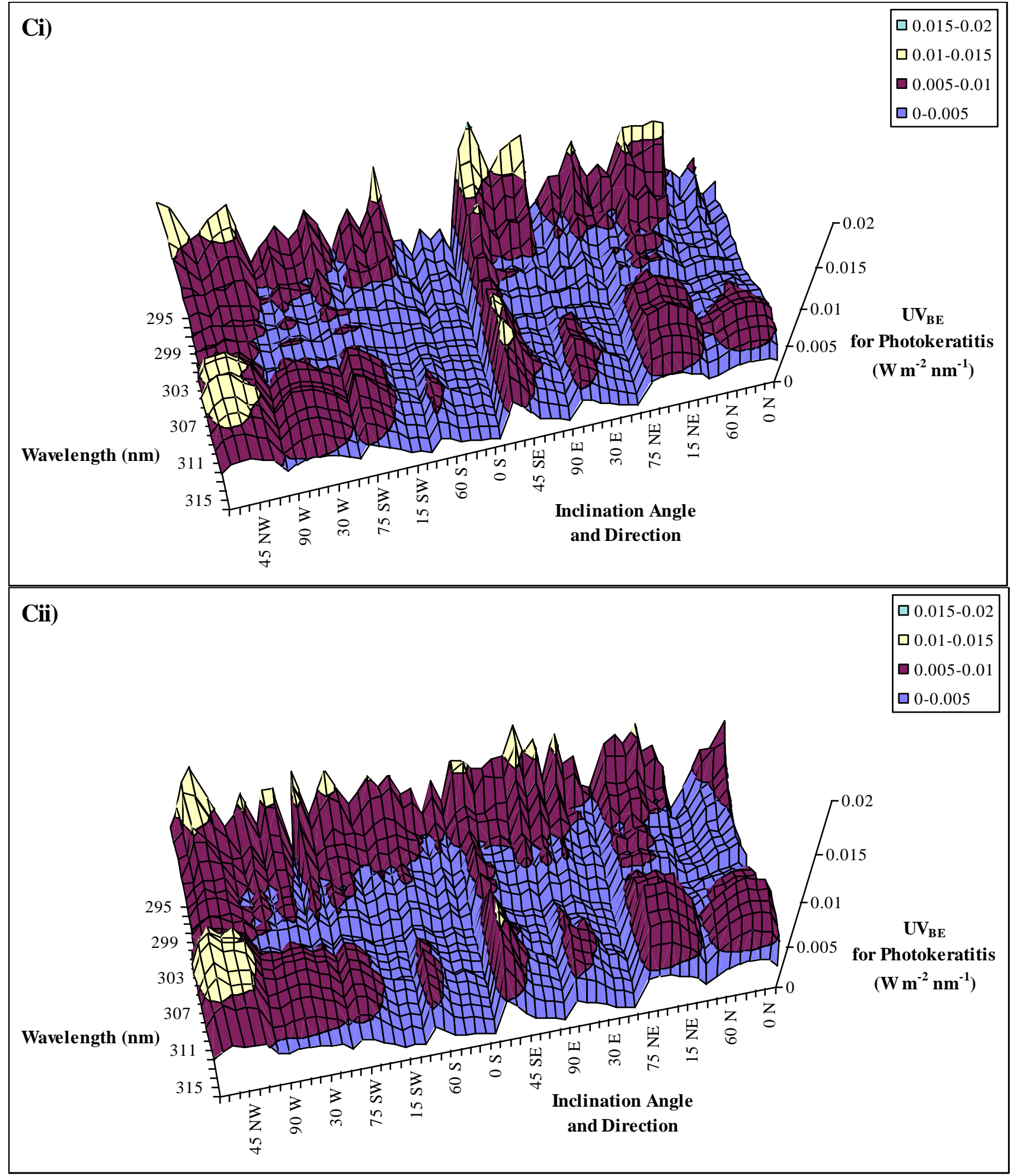

Figure 5: Spectral distributions of the erythemal, photoconjunctivital and photokeratital $\mathrm{UV}_{\mathrm{BE}}$ across the hemispherical domain shown for trial 1 in charts $\mathrm{Ai}$ ),

Bi) and $\mathrm{Ci}$ ) respectively and for trial 2 in charts Aii), Bii) and Cii). 

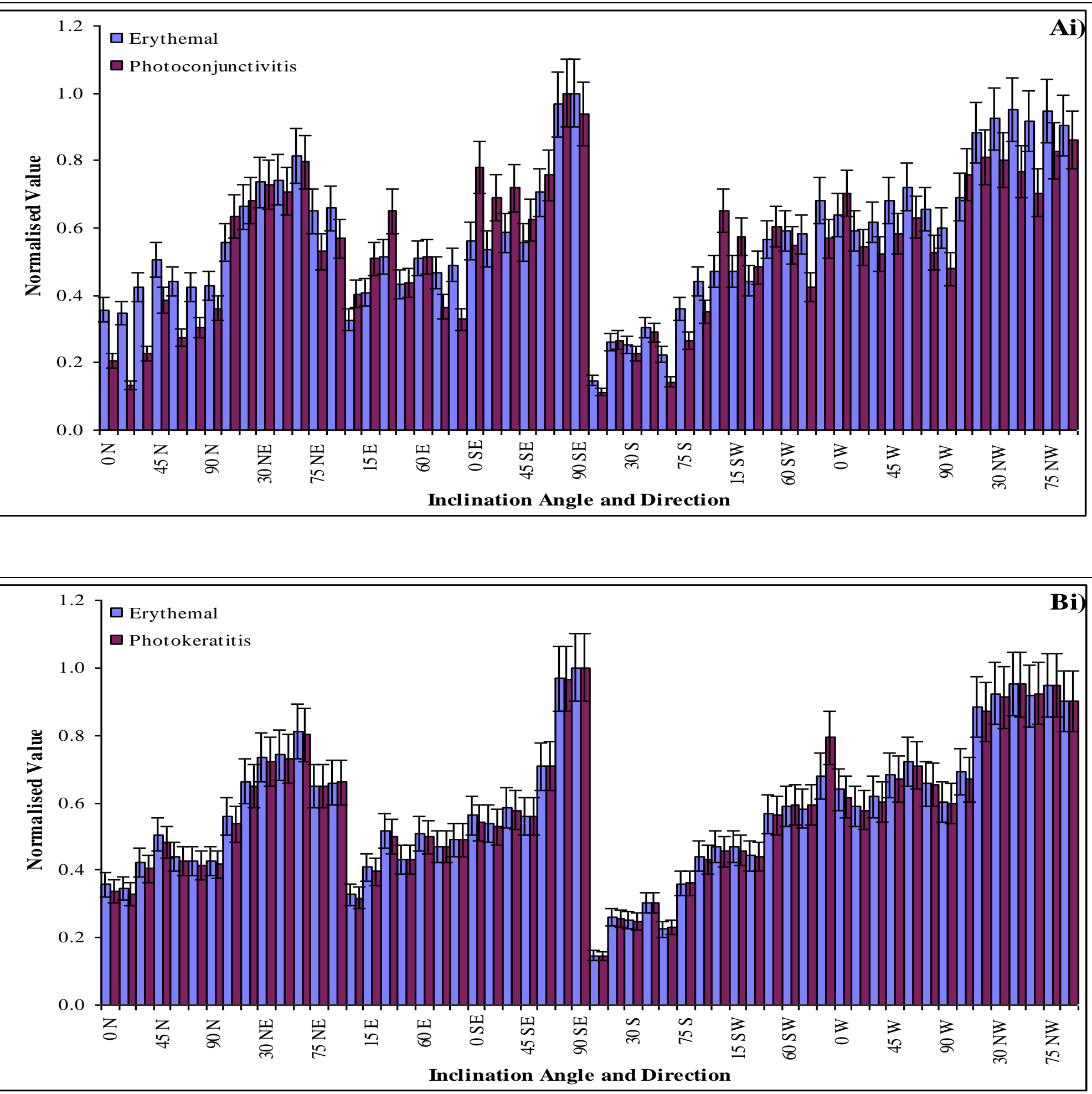

Figure 6: Comparison of the normalised values corresponding to trial $1 \mathrm{for} \mathrm{Ai}$ ) the erythemal and photoconjunctivital response and $\mathrm{Bi}$ ) the erythemal and photokeratital response. The inclination angle is measured in degrees. 
